\title{
Experimental Study of the Human Ability to Deliberately Excite a Flexible Floor
}

\author{
C. Schwartz \\ LAMH, Laboratoire d'Analyse du Mouvement Humain, University of Liege, Belgium
}

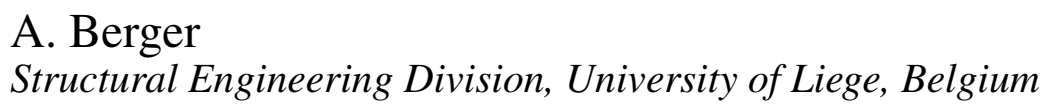

O. Brüls

LAMH, Laboratoire d'Analyse du Mouvement Humain, University of Liege, Belgium

Department of Aerospace and Mechanical Engineering, University of Liege, Belgium

J.-L. Croisier \& B. Forthomme

LAMH, Laboratoire d'Analyse du Mouvement Humain, University of Liege, Belgium

Department of Motivity Sciences, University of Liege, Belgium

V. Denoël

LAMH, Laboratoire d'Analyse du Mouvement Humain, University of Liege, Belgium

Structural Engineering Division, University of Liege, Belgium.

\begin{abstract}
Human-structure interactions are not completely understood yet. This paper presents an experimental study focusing on the interaction between a human and a flexible support, with a major aim to better understand how the behavior of a human subject, bouncing on a flexible structure, adapts to the support motion. Exploratory experiments are undertaken with a 7m-span timber footbridge tested in the Human Motion Analysis Laboratory of the University of Liege. The movements of the coupled system composed of the subject and the footbridge are recorded by Motion Capture technology; the subject's 3-D body motion is defined by three important angles: ankle, knee and hip. The interaction between the subject and the footbridge is also quantified by means of force platform measurements. The experiments indicate an influence of the support amplitude and natural frequency on the phase shift between the oscillatory floor motion and the three angles characterizing the motion.
\end{abstract}

\section{INTRODUCTION}

Vandalism is coined as a specific kind of footbridge loading characterized by the intentional and wellcoordinated action of one or several persons, moving their own body with the sole aim to increase the structural vibration level to a maximum value. The term vandalism actually covers several kinds of action (Zivanovic S. 2005), among which only bouncing sometimes also referred to as bobbing or yoggingis considered in this paper. It consists in a near harmonic knee-extension motion at a frequency equal to, or as close as possible to, a structural natural frequency, which brings the structure into resonance. Of major importance is the reaction force under the vandal's feet, i.e. the basic information required for the structural design of the footbridge since it may be seen as an external loading from the designer's point of view. A proper estimation of this force and of the resulting structural response allows estimation of occurrence of possible harmful vibrations which could, for instance, result in negative vertical reaction forces at the abutments. Several models of reaction forces resulting from bouncing do exist, but are essentially limited to motionless rigid supports (Duarte E. 2009). Recently, Harrison et al. (Harrison R. E \& Dougill 2006, Harrison R. E. 2008) have studied this humanstructure interaction on flexible supports as a function of a number of parameters including the structural mass and damping. A mass- and damping-dependent reduction of the reaction force was observed, while approaching resonance. In contrast with existing lit- 
erature which mainly relies on the body motion description as a single degree-of-freedom system, experimental results reported in this paper include the measurement of three representative body motion angles as well as the floor reaction force and the structural response. The paper is divided into a short description of the experimental setup, a presentation of the main results, followed by the proposition of a simple loading model.

\section{DESCRIPTION OF THE EXPERIMENTAL SETUP}

\subsection{The footbridge}

A toy footbridge composed of two timber beams with a cross-section of $75 \times 225 \mathrm{~mm}$ is designed to behave as a simply supported span of $6.7 \mathrm{~m}$. The two main beams are covered by oriented strand board (OSB) screwed on top of them. This bare structure is ballasted with steel load in order to tune the natural frequency in the range $[2.4 ; 4.2] \mathrm{Hz}$, see Fig. 1 . These additional dead loads are securely fastened to the structure in order to hinder any relative vertical motion. During the experiment, the vandal stands at midspan, where a force platform is installed in order to record the reaction forces. The structure responds in its fundamental vibration mode.
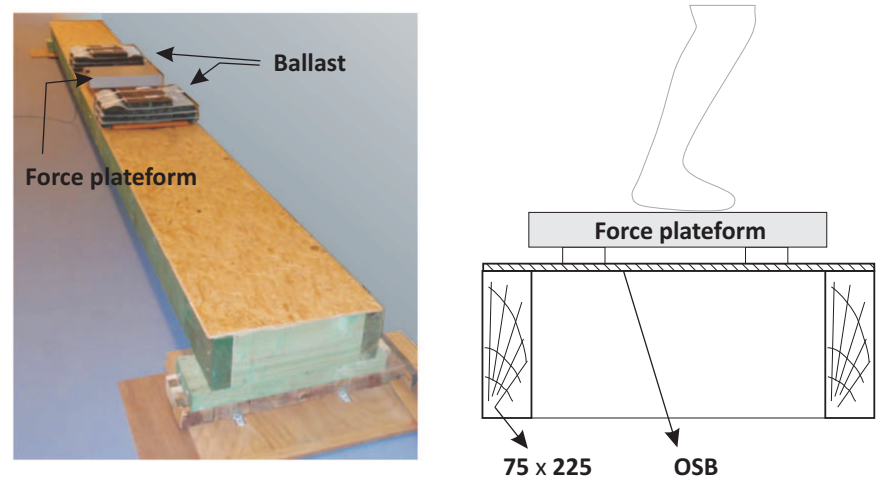

Figure 1: Picture of the toy footbridge, with a force platform at mid-span. Laboratoire d'Analyse du Mouvement Humain (LAMH).

\subsection{Instrumentation of the Human-Structure System}

The instrumentation is performed with a Motion Capture system composed of cameras, markers and the CODAmotion software. The system localizes in space the diodes (markers) placed in various strategical points of the investigated system. Figure 2 shows a snapshot of the planar model constructed with rigid body segments connecting the measured joints, as well as a picture of the vandal in a similar position. Essentially instrumentation of two corners of the force plate allow estimation of the structural motion at mid-span while five anatomical references, namely the great toe, the ankle, the knee, the hip and the

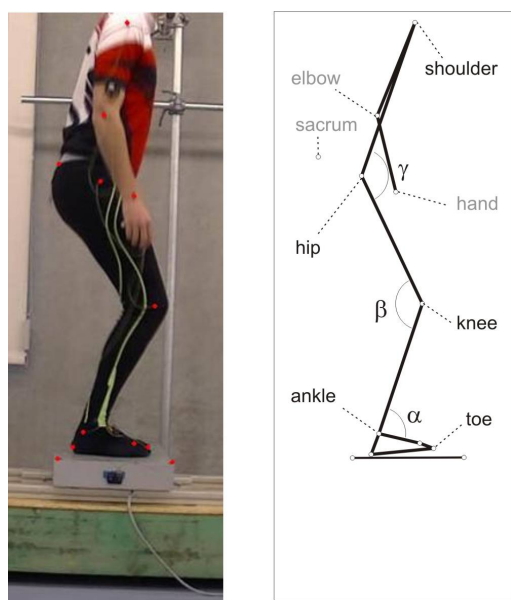

Figure 2: Markers location and reconstructed model with rigid body segments. (Red dots in the picture indicate the full set of measured anatomical references - only four of them are used to define the kinematic angles)
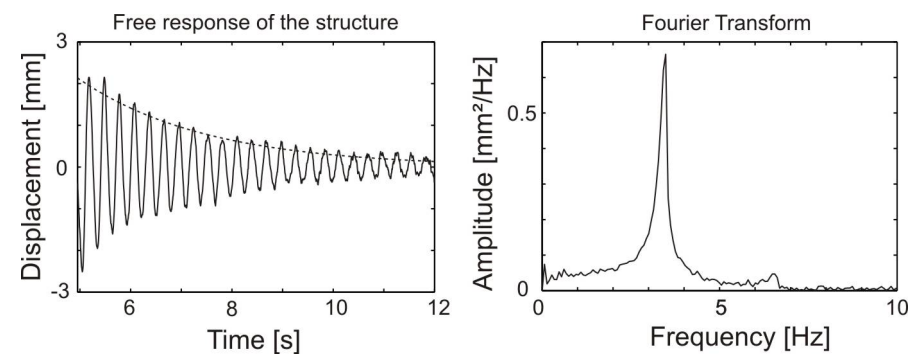

Figure 3: Free-response of the footbridge in the $3.4 \mathrm{~Hz}-$ configuration: time series and Fourier transform. Estimated damping ratio is $2 \%$.

shoulder, provide information about the posture during the excitation. Because the body motion is studied in a vertical plane, these four anatomical references define three kinematic angles $\alpha, \beta$ and $\gamma$, that describe the motion precisely enough.

The reaction force is acquired with a force platform Kistler, $9281 \mathrm{EA}$ which is firmly fastened to the footbridge in order not to disturb the subject's motion. The recorded data are logged and properly synchronized between the markers and the force platform with the software CODAmotion, V6.79.2. The force platform data is sampled at $1000 \mathrm{~Hz}$ and immediately downsampled to match the $200 \mathrm{~Hz}$ sampling frequency of the markers for further postprocessing.

\subsection{Considered Configurations}

Five configurations are considered. Each of them corresponds to a different ballast and thus to a different natural frequency. The effective natural frequency involving the coupled human-structure system is slightly less than the natural frequency of the ballasted structure itself, see Tab. 1. Figure 3 represents the free response of the beam in configuration 3 , with the corresponding frequency spectrum. Such free decay responses are used to identify a damping ratio of $2 \%$ in each configuration. 
Table 1: Modal masses $m$, natural frequencies $f$ of the ballasted structure and natural frequencies $f_{\text {eff }}$ of the human-structure system

\begin{tabular}{|c|c|c|c|c|c|c|}
\hline Configuration & 1 & 2 & 3 & 4 & 5 & \\
\hline$m$ & 1154 & 811 & 615 & 412 & 275 & $\mathrm{~kg}$ \\
\hline$f$ & 2,4 & 2,8 & 3,4 & 3,9 & 4,6 & $\mathrm{~Hz}$ \\
\hline$f_{\text {eff }}$ & 2,3 & 2,8 & 3,2 & 3,7 & 4,2 & $\mathrm{~Hz}$ \\
\hline
\end{tabular}

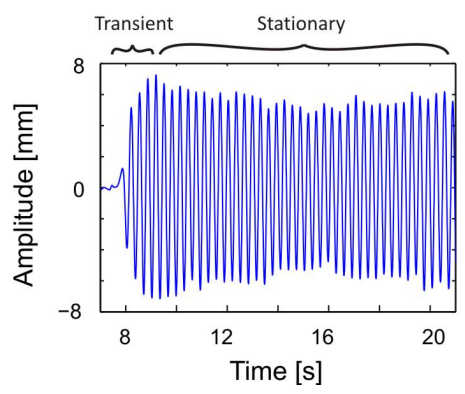

Figure 4: Vertical displacement of the flexible floor, measured with respect to the static deflection of the footbridge under the own weight of the experimenter.

\subsection{Test instructions and measurement protocol}

The results presented next are obtained with a unique subject weighting $87 \mathrm{~kg}$, male, aged 23 . The sole test instruction is to bring the structure into resonance as fast as possible, while keeping heels in contact with the support, and to hold a constant excitation level during at least 15 seconds. A typical response signal thus features two distinct parts: a short transient regime followed by a stationary response with a somewhat constant amplitude.

Tests are reproduced 20 times targeting various levels of amplitude in this stationary regime (slight, weak, medium, large, very large), which is feasible because the sole experimenter was trained and could judge how much energy should be imparted in the test, before it starts.

These two regimes are illustrated in Figure 4, with an example of the displacement of the flexible support, measured with the opto-electronic system, and expressed with respect to the static deflection under the own weight of the test subject.

\section{RESULTS}

\subsection{Overview of a typical result}

Figure 5 represents the displacement, velocity and acceleration of the footbridge in configuration 3 . The transient phase is apparently as short as 1 second. This is by no means much shorter than the transient phase associated with a linear time-invariant system of natural frequency $3.2 \mathrm{~Hz}$ and damping ratio $2 \%$ $\left(t_{r} \simeq 1 /(3.2 \cdot 0.02)=15 \mathrm{~s}\right)$, which evidences the existence of a strong human-structure interaction, reductio ad absurdum.

The corresponding time evolution of the reaction force is represented in Figure 6, together with the phase shift between the force and the structural displacement. The typical profile of the force further il-
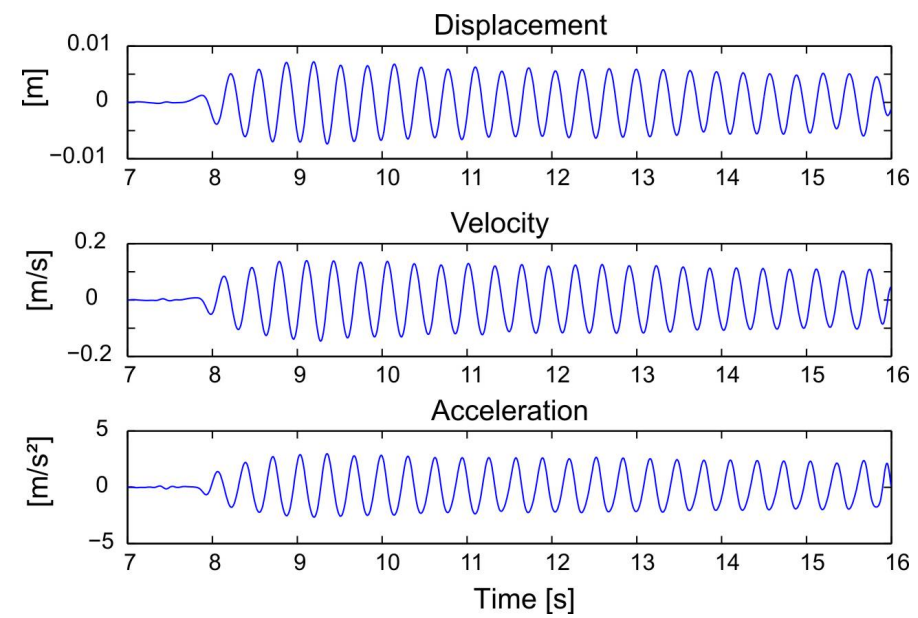

Figure 5: Typical response of the footbridge in configuration 3.
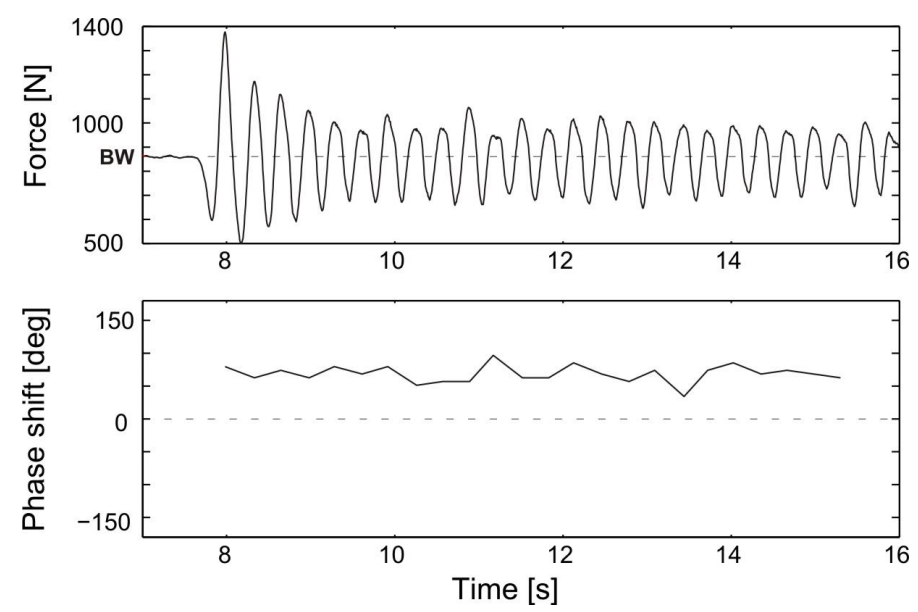

Figure 6: Force reaction and phase shift between force reaction and structural displacement.

lustrates the shortness of the transient phase. It systematically starts with a decreasing magnitude, down to half of the body weight (BW), as the subject pumps down, lowering his center of mass - this corresponds to a decrease of the three angles $\alpha, \beta$ and $\gamma-$. The structural motion is thus initiated upward. Then the subject sets forth, with a knee-bending motion, which results in a significantly increased reaction force, up to 1.6 times the body weight and triggers the structure oscillations. A couple of such pumping cycles is sufficient to reach the steady regime.

A key event in this human-structure interaction is the sensibility of the test subject to adequately identify the right instant when to set forth (around $7.85 \mathrm{~s}$ in this example). In this particular test setup, the pump-down phase results in a perceptible motion $(v \simeq 1 \mathrm{~mm} / \mathrm{s})$ because the potential energy stored in the footbridge under the full body weight transforms into a significant kinetic energy as the reaction force drops to half of the body weight.

Once the stationary regime is achieved, the phase shift between the reaction force and the structural displacement oscillates with slight amplitudes around approximately $70^{\circ}$.

Figure 7 shows the time evolution of the kinematic angles $\alpha, \beta$ and $\gamma$, as well as their phase shift with respect to the structural displacement. Starting from a 

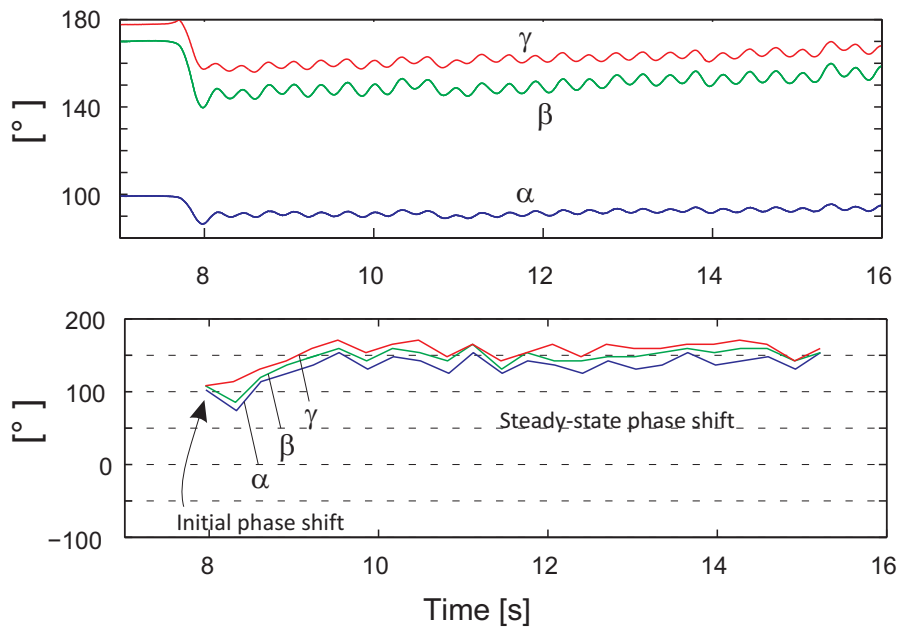

Figure 7: Kinematic angles and phase shift between them and the structural displacement.

more or less vertical rest position, the subject bends his knees, identifies the structural natural frequency from the weak initial motion and performs a quasiperiodic motion. A couple of cycles only is enough for the subject to adapt his excitation frequency, amplitude and delay in order to maximize the structural response. The slight long-term drift in the kinematic angles indicates a slow change of the posture, which may be attributed to the weariness of the subject. Average phase shifts in the steady regime $\bar{\alpha}=138^{\circ}$, $\bar{\beta}=150^{\circ}$ and $\bar{\gamma}=157^{\circ}$ show that these angles are all a phase ahead of the structural displacement. Furthermore, the small advance of the chest-leg angle $\gamma$ indicates that the human motion seems to be initiated from the chest to the knees, and then feet.

Actually, trajectories of the center of mass of the subject (estimated with a sensor located on the sacrum) indicate a kind of quasi-periodic elliptical motion, at least in cases where resonance is achieved $2.4 \mathrm{~Hz}, 2.8 \mathrm{~Hz}$ and $3.2 \mathrm{~Hz}$, see Figure 8 , while the motion is rather chaotic for larger natural frequencies.

Interestingly, the phase shifts between kinematic angles and the structural motion seem to witness a longer transient regime, as it takes from 8 s to $9 \mathrm{~s}$ before the phase shifts between kinematic angles and the structural displacement stabilize. For this reasons, results of the parametric study are analyzed next in the light of two different shifts, the initial phase shift which is measured as the force starts increasing and the steady-state phase shift which corresponds to the long term phase shift, see Figure 7.

\subsection{Parametric study}

Figure 9 shows the initial phase shift for each measurement and for each angle. The black line connects average results. Despite a significant scatter in the data, this diagram shows a clear trend: initial phase shift of angles $\beta$ and $\gamma$ roughly exhibit an augmentation rate of about $30^{\circ} / \mathrm{Hz}$, with a larger initial phase shift for $\gamma$. The ensemble average for angle $\alpha$ is approximately equal to $100^{\circ}$ for frequencies equal to
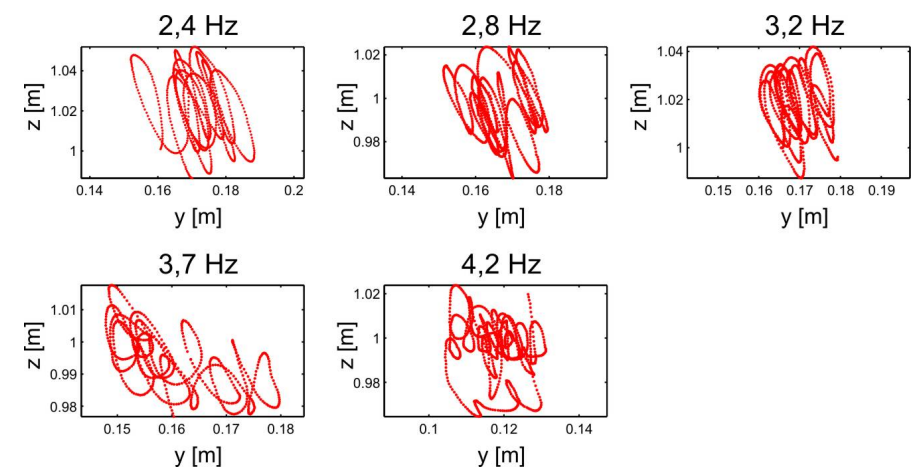

Figure 8: Trajectories of the centre of mass of the subject, for various natural frequencies of the support. While an quasiperiodic motion is observable for $2.4 \mathrm{~Hz}, 2.8 \mathrm{~Hz}$ and $3.2 \mathrm{~Hz}$, the response for $3.7 \mathrm{~Hz}$ and $4.2 \mathrm{~Hz}$ is rather chaotic.
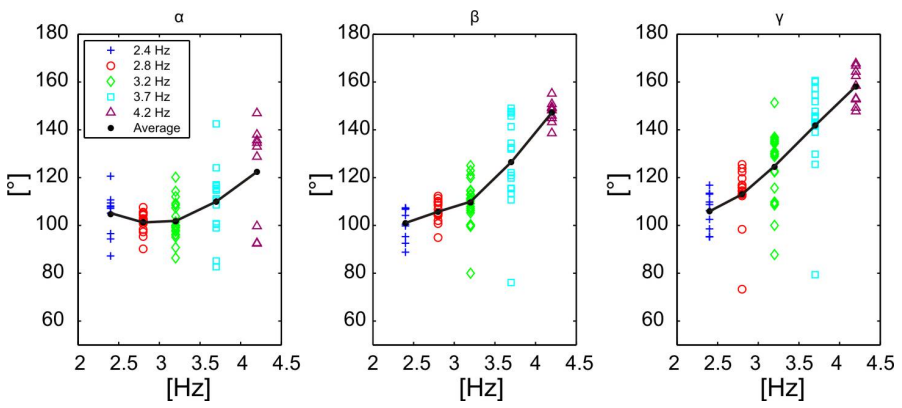

Figure 9: Initial phase shifts for various natural frequencies of the footbridge.

$2,4 \mathrm{~Hz}, 2,8 \mathrm{~Hz}$ and $3,2 \mathrm{~Hz}$ and increases up to $120^{\circ}$ for higher natural frequencies. Also, the initial phase shift associated with $\alpha$ is smaller than the phase shifts associated with $\beta$ and $\gamma$, which suggests that the motion is well initiated from top to bottom, which generalizes what was observed for configuration 3 .

Steady-state phase shifts are reported in Figure 10 as a function of the maximum displacement of the footbridge on the left, and as a function of the natural frequency on the right. Again, despite a significant scatter in the data, there is a clear trend indicating that the higher the natural frequency, the larger the phase shift. More precisely the rates of increase of angles $\alpha, \beta$ and $\gamma$ are approximately equal to $8^{\circ} / \mathrm{Hz}, 16^{\circ} / \mathrm{Hz}$ and $10^{\circ} / \mathrm{Hz}$, respectively.

Figure 11 represents the phase shift between the reaction force and the footbridge displacement, as a function of the maximum structural displacement (left) and of the natural frequency (right). While the optimum phase shift between a harmonic force and the response of a single-degree-of-freedom that maximizes the energy pumping into the system is, from a mechanical viewpoint, equal to $90^{\circ}$, the human brainbody controller is not able to adjust to this optimality of control. Figure 11 indicates that this optimum delay is only materialized when vibration amplitudes of the footbridge are small. Even for moderate structural displacements and accelerations, this optimum phase shift is hardly hold by the subject. Indeed, the time delay required for communication between the brain and the sensorimotor activities, the treatment of information generated by sensory organs, the identification 

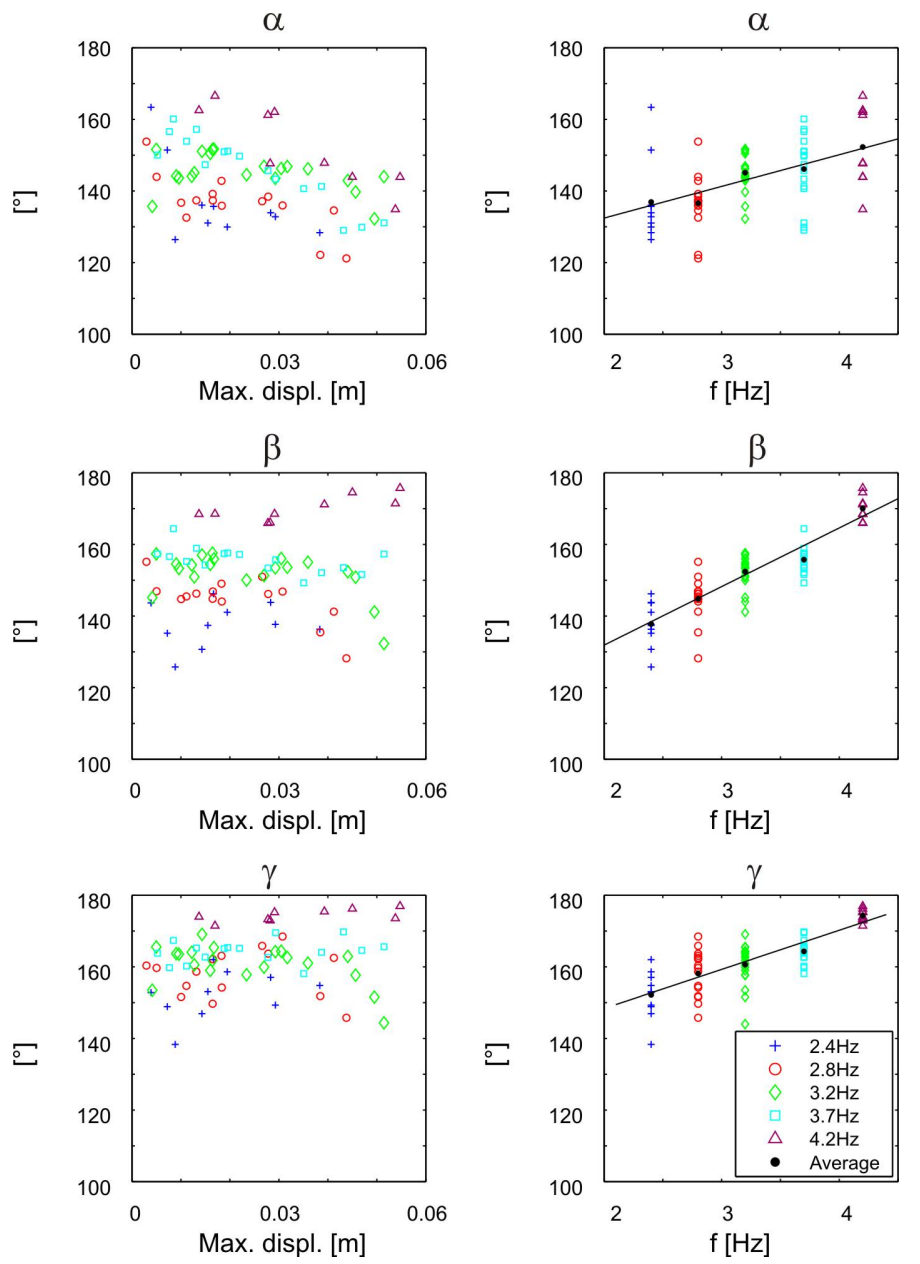

Figure 10: Steady-state phase shift between the kinematic angles and the structural displacement represented as a function of the maximum displacement (left) and of the structural natural frequency (right).
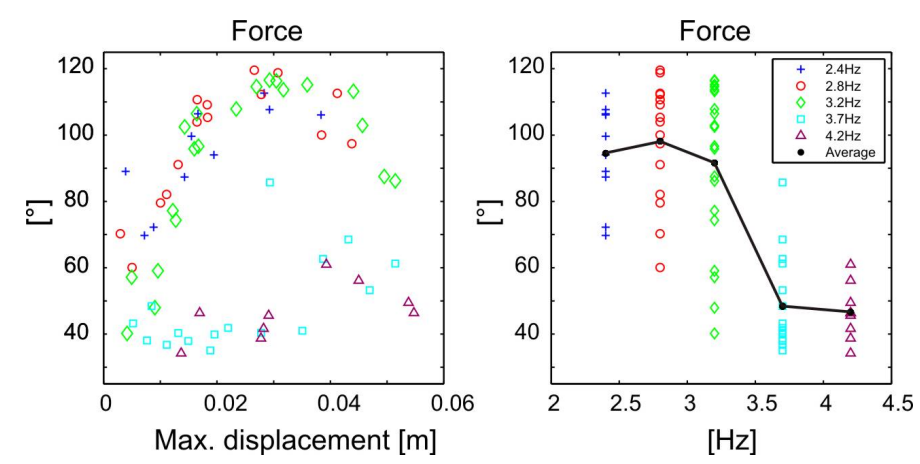

Figure 11: Steady-state phase shift between the force and the structural displacements represented as a function of the maximum displacement (left) and of the structural natural frequency (right). of a natural frequency and finally the muscle activation make it impossible for a human being to adjust to this mechanical optimum.

For natural frequencies of $2.4 \mathrm{~Hz}, 2.8 \mathrm{~Hz}$ and 3.2 $\mathrm{Hz}$, the phase shift seems to stabilize around $110^{\circ}$ for displacements in the range $1.5-4.5 \mathrm{~cm}$. For smaller amplitudes, large scatterness indicates that the subject has difficulties in feeling a sufficient level of vibration, and in adapting its motion to that frequency; he thus provides the impulse with a smaller delay $\left(80^{\circ}\right)$.

A similar explanation may justify the short delay $\left(40^{\circ}-60^{\circ}\right)$ for frequencies of motion equal to $3.7 \mathrm{~Hz}$ and $4.2 \mathrm{~Hz}$. In this case, the frequency of the motion is too high and the subject is not able to move that fast. The existence of this discontinuity in the response, when considered as a function of the natural frequency of the footbridge, is however not observable in the body posture, i.e. in angles $\alpha, \beta$ and $\gamma$, see Figure 10. This indicates that, despite an apparently similar body behaviour, the subject is not able to impose an optimum phase shift in the force.

\section{CONCLUSIONS}

This experimental study of the human-structure interaction in a bouncing motion has objectivized a series of facts. First, the human body motion parametrized in this study with three kinematic angles, which is a major innovation of this experimentseems to be initiated from chest to feet. Second, there are basically two conditions in which the subject is not able to tune his excitation frequency on the structural motion. On the one hand, for small amplitudes of vibration, the subject is not capable of identifying the natural frequency and the phase of the structural loading, which makes it impossible for him to properly synchronize. On the other hand, for large frequencies of support oscillations, the subject has not the physical reactivity to excite the footbridge fast enough, which finally translates into a chaotic uneffective motion. This is presumably due to the communication with the brain feedback, and the delay in the muscle activation. In both cases, this results in a reaction force, i.e. the force transmitted from the vandal to the structure, that is not optimally synchronized with the support motion, and consequently turns into small amplitudes of vibration. Actually in these cases, the phase shift between the support motion and the reaction force may even drop down to $40^{\circ}-60^{\circ}$ in the latter case.

The results obtained with a single subject indicate the existence of a critical natural frequency (in the range $[3.2 ; 3.7] \mathrm{Hz}$ for this particular subject) beyond which synchronization of the force and structural displacement is not achieved. As perplexing as it may appear, this discontinuity in the reaction force does not replicate in the steady-state phase shift between the kinematic angles and the support motion. Additional experimental work, involving EMG in order to 
survey muscle activity, will be carried out in a near future in order to clarify this point.

\section{ACKNOWLEGMENT}

The authors would like to acknowledge the Federation Wallonie-Bruxelles for their support in the development of the laboratory (http://www.lamh.ulg.ac.be)

\section{REFERENCES}

Duarte E., J. T. (2009). Action of individual bouncing on structures. Journal of Structural Engineering, 818-827.

Harrison R. E, Wright, J. R. \& J. W. Dougill (2006). Video monitoring of human-structure interaction for bouncing on a flexible structure. In Proc., 24th Int. Modal Analysis Conf. (IMACXXIV), Society for Experimental Mechanics SEM, Bethel, Conn.

Harrison R. E., Yao S., W. J. R. P. A. R. P. (2008). Human jumping and bobbing forces on flexible structures: Effect of structural properties. Journal of Engineering Mechanics, 663-675.

Zivanovic S., Pavic A., R. P. (2005). Vibration serviceability of footbridges under human-induced excitation: a literature review. Journal of Sound and Vibration 1-74, 279-. 